Pacific Journal of Mathematics

ON STRONGLY NONLINEAR ELLIPTIC VARIATIONAL

( 


\section{ON STRONGLY NONLINEAR ELLIPTIC VARIATIONAL INEQUALITIES}

\section{BuI AN ToN}

The existence of bounded solutions of nonlinear elliptic variational inequalities is shown. The nonlinear second order elliptic operator involved has at most an exponential growth in $u$ and a polynomial growth in $D u$. The regularity of the solutions is studied.

Let $K$ be a closed convex subset of a reflexive Banach space $V$ and let $A$ be a pseudo-monotone coercive operator from $V$ into $V^{*}$. Then for any $f$ in $V^{*}$ it is known that there exists $u$ in $K$ such that:

$$
(A u-f, v-u) \geqslant 0 \quad \text { for all } v \text { in } K \text {. }
$$

The existence of solutions of nonlinear elliptic variational inequalities has been shown by Brezis [1], Browder [4], Lions-Stampacchia [10] and others. The regularity of the solutions when $A$ is a linear second order elliptic operator written in divergence form has been studied by Brezis [2], Lewy-Stampacchia [8], Lions [9] and an abstract regularity result has been obtained by Brezis-Stampacchia [3] when $A$ is nonlinear.

It is the purpose of this paper to show the existence of bounded solutions of nonlinear variational inequalities when $A$ is a pseudomonotone coercive operator defined on $V \cap L^{\infty}(G)$ and mapping $V \cap L^{\infty}(G)$ into $V^{*} . \quad V$ is a given closed subspace of $W^{1, p}(G)$ with $W_{0}^{1, p}(G) \subset V \subset$ $W^{1, p}(G)$. The nonlinear elliptic operator $A$ may have an exponential growth in $u$ and a polynomial growth in $D u$. Abstract existence theorems are proved in $\S 2$. The applications and the regularity of the solutions are studied in $\S 3$. The notations and the basic assumptions are given in $\S 1$.

1. Let $G$ be a bounded open subset of $R^{n}$ with a smooth boundary $\partial G$. Set: $D_{j}=\partial / \partial x_{j}, j=1, \cdots, n$ and

$$
W^{1, p}(G)=\left\{u: u \quad \text { in } \quad L^{p}(G), D_{j} u \quad \text { in } \quad L^{p}(G), j=1, \cdots, n\right\} .
$$

$W^{1, p}(G)$ is a real reflexive separable Banach space with the norm:

$$
\|u\|_{1, p}=\left\{\|u\|_{L}^{p} p_{(G)}+\sum_{j=1}^{n}\left\|D_{j} u\right\|_{L}^{p} p_{(G)}\right\}^{1 / p} ; 2 \leqslant p<\infty .
$$

$W_{0}^{1, p}(G)$ is the completion of $C_{0}^{\infty}(G)$, the family of all infinitely differentiable functions with compact support in $G$, in the $\|\cdot\|_{1, p}$ norm. 
Let $V$ be a closed linear subspace of $W^{1, p}(G)$ with $W_{0}^{1, p}(G) \subset V \subset$ $W^{1, p}$ and let $H=L^{2}(G)$. The pairing between $V$ and its dual $V^{*}$ is denoted by $(\cdot, \cdot)$.

Let $W$ be a closed linear subspace of $W^{1, p+n}(G)$ with $W_{0}^{1, p+n}(G) \subset$ $W \subset W^{1, p+n}(G)$. The Sobolev imbedding theorem gives $W \subset C(\mathrm{cl} G)$. The pairing between $W$ and $W^{*}$ is denoted by $((\cdot, \cdot))$. Throughout the paper we shall assume:

(a) $W \subset V$.

(b) If $u$ is in $W$ then $|u|^{s-2} u$ is also in $W$ for all $s$ with $2 \leqslant$ $s<\infty$. The assumption is verified if $V=W_{0}^{1 . p}(G), W=W_{0}^{1, p+n}(G)$ and if $V=W^{1, p}(G)$ with $W=W^{1, p+n}(G)$.

$C^{0, \alpha}(G), 0<\alpha<1$, is the family of all functions $u$ which are Hölder-continuous with exponent $\alpha$ on any compact subset of $G$.

Set:

$$
F=V \cap L^{\infty}(G)
$$

In this paper nonlinear operators $A(u, v)$ mapping $F \times V$ into $V^{*}$ satisfying the following assumption are considered.

Assumption (I): (i) $A(u, v)$ maps bounded sets of $F \times V$ into bounded sets of $V^{*}$.

(ii) If $u_{n} \rightarrow u$ weakly in $V, u_{n} \rightarrow u$ in the weak*-topology of $L^{\infty}(G)$ and $\lim \sup \left(A\left(u_{n}, u_{n}\right), u_{n}-u\right) \leqslant 0$ then:

$$
\begin{array}{r}
\lim \inf \left(A\left(u_{n}, u_{n}\right), u_{n}-v\right) \geqslant(A(u, u), u-v) \\
\text { for all } v \text { in } V .
\end{array}
$$

The pseudo-monotone operators from $V$ into $V^{*}$ satisfy all the conditions of Assumption (I).

Let $A(u, v)$ be the operator defined by:

$$
\begin{aligned}
(A(u, v), w)= & \sum_{j=1}^{n} \int_{G}(1+c(x) \exp u)\left|D_{j} v\right|^{p-2} D_{j} v D_{j} w d x \\
& +\int_{G} b(x) u \exp u \cdot w d x
\end{aligned}
$$

$u$ is in $F, v, w$ in $V, c(x)$ and $b(x)$ are two nonnegative bounded functions on $G . A(u, v)$ is the prototype of operators considered in this paper. It is not difficult to check that $A(u, v)$ satisfies all the conditions of Assumption (I).

In the proof of the existence theorems we need the following auxiliary nonlinear monotone operator:

$$
A_{2} v=-\sum_{j=1}^{n} D_{j}\left(\left|D_{j} v\right|^{p+n-2} D_{j} v\right)+v
$$


2. In this section the existence of bounded solutions of nonlinear elliptic variational inequalities is shown.

The following theorem gives a generalization of a result of Browder [4], Hartman-Stampacchia [6] and others when $V$ is a closed linear subspace of $W^{1, p}(G)$.

THEOREM 1. Let $A(u, v)$ be a nonlinear operator mapping $F \times$ $V$ into $V^{*}$ and satisfying Assumption (I). Let $K$ be a closed convex subset of $V$ with $0 \in K$ and let $\beta$ be the penalty operator associated with $K$. Suppose that:

(i) $(A(u, u), u) \geqslant c\|u\|_{V}^{p}$ for all $u$ in $F$.

(ii) $\left(A(u, u),|u|^{s-2} u\right) \geqslant c\|u\|_{L^{s}(G)}^{s}$ for all $u$ in $W$ and all $s$ with $2 \leqslant s<\infty$. The positive constant $c$ is independent of $s$.

(iii) $\left(\beta(u),|u|^{s-2} u\right) \geqslant 0$ for all $u$ in $W$ and all $s, 2 \leqslant s<\infty$. Then for any $f$ in $L^{\infty}(G)$ there exists $u$ in $F \cap K$ such that:

$$
(A(u, u)-f, v-u) \geqslant 0 \quad \text { for all } v \text { in } K \text {. }
$$

Theorem 2. Let $A(u, w)$ be as in Theorem 1 and let $K$ be a closed convex subset of both $V$ and $W$ with $0 \in K$. Let $\beta$ be the penalty operator associated with $K$ considered as a subset of $W$. Suppose all the hypotheses of Theorem 1 are satisfied with (iii) replaced by:

$$
((\beta(u), u)) /\|u\|_{w} \longrightarrow+\infty \text { as }\|u\|_{W} \longrightarrow+\infty \text {. }
$$

Then for any $f$ in $L^{\infty}(G)$ there exists $u$ in $F \cap K$ such that:

$$
(A(u, u)-f, v-u) \geqslant 0 \quad \text { for all } v \text { in } K \text {. }
$$

For a smaller class of nonlinear operators $A(u, v)$ we have the following theorem which extends a result of Dubinskii [5].

THEOREM 3. Let $A(u, v)$ be a nonlinear operator mapping bounded sets of $L^{\infty}(G) \times V$ into bounded sets of $V^{*}$. Suppose that: in $V$.

(i ) $\left(A(u, v)-A(u, w), v-w \geqslant 0\right.$ for all $u$ in $L^{\infty}(G)$ and all $v, w$

(ii) For fixed $u$ in $L^{\infty}(G), A(u, \cdot)$ is continuous from the strong topology of $V$ to the weak topology of $V^{*}$.

(iii) For fixed $v$ in $V$, if $u_{n} \rightarrow u$ a.e. on $G$ and $u_{n} \rightarrow u$ in the weak*-topology of $L^{\infty}(G)$ then: $A\left(u_{n}, v\right) \rightarrow A(u, v)$ in $V^{*}$.

(iv) $\left(A\left(|u|^{s-2} u, u\right), u\right) \geqslant c\|u\|_{V}^{p}$ for all $u$ in $W$ and all $s$ with $1<$ $s<\infty$.

(v) $\left(A\left(|u|^{s-2} u, u\right),|u|^{r-2} u\right) \geqslant c\|u\|_{L^{r+s-2}(G)}^{r+s-2}$ for all $u$ in $W$ with $1<s<\infty$ and $2 \leqslant r<\infty$. The constant $c$ is independent of $r$.

Then for any $f$ in $L^{\infty}(G)$ there exists $u$ in $L^{\infty}(G)$ with $|u|^{x-2} u$ in $V$ such that: 


$$
A\left(u,|u|^{\alpha-2} u\right)=f .
$$

$\alpha$ is any number with $1<\alpha<\infty$.

Proof of Theorem 1. (1) Let $0<\eta<\varepsilon<1$ and let $\mathscr{A}(u, v)$ be the nonlinear operator:

$$
\mathscr{A}(u, v)=\eta A_{2} v+A(u, u)+\varepsilon^{-1} \beta(u) .
$$

$\mathscr{A}(u, v)$ maps bounded sets of $W \times W$ into bounded sets of $W^{*}$ and is coercive. Being the sum of a monotone operator and a pseudomonotone operator, $\mathscr{A}$ is a pseudo-monotone operator from $W$ into $W^{*}$. It follows from the theory of coercive pseudo-monotone operators that there exists $u_{\varepsilon \eta}$ in $W$, solution of the equation:

$$
\eta A_{2} u_{\varepsilon \eta}+A\left(u_{\varepsilon \eta}, u_{\varepsilon \eta}\right)+\varepsilon^{-1} \beta\left(u_{\varepsilon \eta}\right)=f .
$$

It is clear that:

$$
\eta\left\|u_{\varepsilon \eta}\right\|_{W}^{p+n}+\left\|u_{\varepsilon \eta}\right\|_{V} \leqslant C
$$

$C$ is a constant independent of both $\varepsilon$ and $\eta$.

Since $u_{\varepsilon \eta}$ is in $W,\left|u_{\varepsilon \eta}\right|^{s-2} u_{\varepsilon \eta}$ lies also in $W$ for all $s$ with $2 \leqslant s<\infty$. Thus:

$$
\begin{aligned}
\eta\left(\left(A_{2} u_{\varepsilon \eta},\left|u_{\varepsilon \eta}\right|^{s-2} u_{\varepsilon \eta}\right)\right)+ & \left(A\left(u_{\varepsilon \eta}, u_{\varepsilon \eta}\right),\left|u_{\varepsilon \eta}\right|^{s-2} u_{\varepsilon \eta}\right) \\
+ & \varepsilon^{-1}\left(\beta\left(u_{\varepsilon \eta}\right),\left|u_{\varepsilon \eta}\right|^{s-2} u_{\varepsilon \eta}\right)=\left(f,\left|u_{\varepsilon \eta}\right|^{s-2} u_{\varepsilon \eta}\right) .
\end{aligned}
$$

An elementary computation gives:

$$
\left(\left(A_{2} u_{\varepsilon \eta},\left|u_{\varepsilon \eta}\right|^{s-2} u_{\varepsilon \eta}\right)\right) \geqslant 0 .
$$

From the hypotheses of the theorem, we get:

$$
\left\|u_{\varepsilon \eta}\right\|_{L^{s}(G)} \leqslant C\|f\|_{L^{\infty}(G)} \text {. }
$$

Since $u_{\varepsilon \eta}$ is in $W$ hence in $L^{\infty}(G)$ we may let $s \rightarrow+\infty$. So:

$$
\left\|u_{\varepsilon \eta}\right\|_{L^{\infty}(G)} \leqslant C\|f\|_{L^{\infty}(G)} \cdot
$$

$C$ is a constant independent of both $\varepsilon$ and $\eta$.

(2) From the weak compactness of the unit ball in a reflexive Banach space we obtain by taking subsequences if necessary: $u_{\varepsilon \eta} \rightarrow u_{\varepsilon}$ weakly in $V, u_{\varepsilon \eta} \rightarrow u_{\varepsilon}$ in the weak*-topology of $L^{\infty}(G), \eta^{1 / p+n} u_{\varepsilon \eta} \rightarrow 0$ weakly in $W, A\left(u_{\varepsilon \eta}, u_{\varepsilon \eta}\right) \rightarrow g_{\varepsilon}$ weakly in $V^{*}$ and $\beta\left(u_{\varepsilon \eta}\right) \rightarrow h_{\varepsilon}$ weakly in $V^{*}$ as $\eta \rightarrow 0$.

So:

$$
\left\|u_{\varepsilon}\right\|_{V}+\left\|u_{\varepsilon}\right\|_{L^{\infty}(G)} \leqslant C
$$




\section{Moreover:}

$$
g_{\varepsilon}+\varepsilon^{-1} h_{\varepsilon}=f .
$$

We show that $g_{\varepsilon}=A\left(u_{\varepsilon}, u_{\varepsilon}\right)$ and $h_{\varepsilon}=\beta\left(u_{\varepsilon}\right)$. We have:

$$
\limsup _{\eta \rightarrow 0}\left(A\left(u_{\varepsilon \eta}, u_{\varepsilon \eta}\right)+\varepsilon^{-1} \beta\left(u_{\varepsilon \eta}\right), u_{\varepsilon \eta}\right) \leqslant\left(g_{\varepsilon}+\varepsilon^{-1} h_{\varepsilon}, u_{\varepsilon}\right) .
$$

On the other hand:

$$
\begin{array}{r}
\left(A\left(u_{\varepsilon \eta}, u_{\varepsilon \eta}\right), u_{\varepsilon \eta}-u_{\varepsilon}\right)=\left(A\left(u_{\varepsilon \eta}, u_{\varepsilon \eta}\right)+\varepsilon^{-1} \beta\left(u_{\varepsilon \eta}\right), u_{\varepsilon \eta}-u_{\varepsilon}\right) \\
\quad-\varepsilon^{-1}\left(\beta\left(u_{\varepsilon \eta}\right)-\beta\left(u_{\varepsilon}\right), u_{\varepsilon \eta}-u_{\varepsilon}\right)-\varepsilon^{-1}\left(\beta\left(u_{\varepsilon}\right), u_{\varepsilon \eta}-u_{\varepsilon}\right) .
\end{array}
$$

Taking into account the monotonicity of the penalty operator, we get:

$$
\begin{aligned}
\left(A\left(u_{\varepsilon \eta}, u_{\varepsilon \eta}\right), u_{\varepsilon \eta}-u_{\varepsilon}\right) \leqslant\left(A\left(u_{\varepsilon \eta}, u_{\varepsilon \eta}\right)\right. & \left.+\varepsilon^{-1} \beta\left(u_{\varepsilon \eta}\right), u_{\varepsilon \eta}-u_{\varepsilon}\right) \\
& -\varepsilon^{-1}\left(\beta\left(u_{\varepsilon}\right), u_{\varepsilon \eta}-u_{\varepsilon}\right) .
\end{aligned}
$$

Thus:

$$
\limsup _{\eta \rightarrow 0}\left(A\left(u_{\varepsilon \eta}, u_{\varepsilon \eta}\right), u_{\varepsilon \eta}-u_{\varepsilon}\right) \leqq 0
$$

Assumption (I) gives:

$$
A\left(u_{s}, u_{s}\right)=g_{s} .
$$

It is now easy to show that $h_{\varepsilon}=\beta\left(u_{\varepsilon}\right)$.

(3) Again from the weak compactness of the unit ball in a reflexive Banach space we get by taking subsequences if necessary: $u_{\varepsilon} \rightarrow u$ weakly in $V, u_{\varepsilon} \rightarrow u$ in the weak*-topology of $L^{\infty}(G)$ and $A\left(u_{\varepsilon}\right.$, $\left.u_{\varepsilon}\right) \rightarrow g$ weakly in $V^{*}$ as $\varepsilon \rightarrow 0$.

Since:

$$
A\left(u_{\varepsilon}, u_{\varepsilon}\right)+\varepsilon^{-1} \beta\left(u_{\varepsilon}\right)=f
$$

it is clear that

$$
\beta\left(u_{\varepsilon}\right) \longrightarrow 0 \text { in } V^{*} \text { as } \varepsilon \longrightarrow 0 \text {. }
$$

The penalty operator $\beta$ is a monotone hemi-continuous operator from $V$ into $V^{*}$. A standard argument gives:

$$
\beta(u)=0 \text { i.e., } u \in K \text {. }
$$

We have:

$$
\begin{aligned}
\left(A\left(u_{\varepsilon}, u_{\varepsilon}\right), u_{\varepsilon}-u\right) & =\left(f-\varepsilon^{-1} \beta\left(u_{\varepsilon}\right), u_{\varepsilon}-u\right) \\
& =\left(f, u_{\varepsilon}-u\right)-\varepsilon^{-1}\left(\beta\left(u_{\varepsilon}\right)-\beta(u), u_{\varepsilon}-u\right) .
\end{aligned}
$$

Thus: 


$$
\left(A\left(u_{\varepsilon}, u_{\varepsilon}\right), u_{\varepsilon}-u\right) \leqq\left(f, u_{\varepsilon}-u\right)
$$

Hence:

$$
\limsup _{\varepsilon \rightarrow 0}\left(A\left(u_{\varepsilon}, u_{\varepsilon}\right), u_{\varepsilon}-u\right) \leqq 0 .
$$

Assumption (I) gives:

$$
A(u, u)=g
$$

and

$$
\liminf _{\varepsilon \rightarrow 0}\left(A\left(u_{\varepsilon}, u_{\varepsilon}\right), u_{\varepsilon}-v\right) \geqslant(A(u, u), u-v) .
$$

Let $v \in K$ and we have:

$$
\begin{aligned}
\left(A\left(u_{\varepsilon}, u_{\varepsilon}\right)-f, v-u_{\varepsilon}\right) & =-\varepsilon^{-1}\left(\beta\left(u_{\varepsilon}\right), v-u_{\varepsilon}\right) \\
& =\varepsilon^{-1}\left(\beta(v)-\beta\left(u_{\varepsilon}\right), v-u_{\varepsilon}\right) .
\end{aligned}
$$

So:

$$
\left(A\left(u_{\varepsilon}, u_{\varepsilon}\right)-f, v-u_{\varepsilon}\right) \geqslant 0 \quad \text { for all } v \text { in } K .
$$

Let $\varepsilon \rightarrow 0$ and we obtain:

$$
(A(u, u)-f, v-u) \geqq 0 \quad \text { for all } v \text { in } K .
$$

The theorem is proved.

Proof of Theorem 2. The proof is similar to that of Theorem 1, we shall not reproduce it.

Proof of Theorem 3. (1) Let $0<\varepsilon<1$ and consider the nonlinear operator $\mathscr{A}(u, v)$ defined by:

$$
\mathscr{A}(u, v)=\varepsilon A_{2} v+A\left(|u|^{\alpha^{\prime}-2} u, u\right)
$$

$u$ and $v$ are in $W$ and $1 / \alpha+1 / \alpha^{\prime}=1$.

$\mathscr{A}$ is coercive and maps bounded sets of $W \times W$ into bounded sets of $W^{*}$. Being the sum of a monotone operator and a pseudomonotone operator, $\mathscr{A}$ is pseudo-monotone. Therefore, there exists $v_{\varepsilon}$ in $W$, solution of the equation:

$$
\varepsilon A_{2} v_{\varepsilon}+A\left(\left|v_{\varepsilon}\right|^{\alpha^{\prime}-2} v_{\varepsilon}, v_{\varepsilon}\right)=f .
$$

It is easy to show that:

$$
\varepsilon\left\|v_{\varepsilon}\right\| W_{W}^{p+n}+\left\|v_{\varepsilon}\right\|_{V} \leqslant C .
$$

$C$ is a constant independent of $\varepsilon$. 
Since $v_{\varepsilon}$ is in $W,\left|v_{\varepsilon}\right|^{s-2} v_{\varepsilon}$ lies in $W$ for all $s$ with $2 \leqslant s<\infty$. Thus:

$$
\varepsilon\left(\left(A_{2} v_{\varepsilon},\left|v_{\varepsilon}\right|_{\varepsilon}^{s-2} v_{\varepsilon}\right)\right)+\left(A\left(\left|v_{\varepsilon}\right|^{\alpha^{\prime}-2} v_{\varepsilon}, v_{\varepsilon}\right),\left|v_{\varepsilon}\right|^{s-2} v_{\varepsilon}\right)=\left(f,\left|v_{\varepsilon}\right|^{s-2} v_{\varepsilon}\right) .
$$

An elementary computation shows that the first term of the left hand side of the above equation is positive. It follows from the hypotheses of the theorem that:

$$
\left\|v_{\varepsilon}\right\|_{L^{s+\alpha^{\prime}-2(G)}}^{\alpha^{\prime}-1} \leqslant C\|f\|_{L^{\infty}(G)} .
$$

Since $v_{\varepsilon}$ is in $W$ we may let $s \rightarrow+\infty$. Hence:

$$
\left\|v_{\varepsilon}\right\|_{L^{\infty}(G)} \leqslant M
$$

$M$ is independent of $\varepsilon$.

(2) The weak compactness of the unit ball in a reflexive Banach space gives by taking subsequences if necessary: $v_{\varepsilon} \rightarrow v$ weakly in $V, v_{\varepsilon} \rightarrow v$ in the weak ${ }^{*}$-topology of $L^{\infty}(G), \varepsilon^{1 / p+n} v_{\varepsilon} \rightarrow 0$ weakly in $W$, $\left|v_{\varepsilon}\right| \alpha^{\prime-2} v_{\varepsilon} \rightarrow h$ weakly in $L^{\alpha}(G)$ and

$$
A\left(\left|v_{\varepsilon}\right|^{\alpha^{\prime}-2} v_{\varepsilon}, v_{\varepsilon}\right) \longrightarrow g \text { weakly in } V^{*} \text { as } \varepsilon \longrightarrow 0 .
$$

Since the injection mapping of $V$ into $H$ is compact, the Lebesgue convergence theorem yields:

$$
v_{\varepsilon} \longrightarrow v \text { in } L^{s}(G) \text { for any } s \text { with } 1<s<\infty .
$$

Thus:

$$
\left.\left|v_{\varepsilon}\right|\right|^{\alpha^{\prime}-2} v_{\varepsilon} \longrightarrow|v|^{\alpha^{\prime}-2} v \text { a.e. on } G \text { and } h=|v|^{\alpha^{\prime}-2} v \text {. }
$$

We have:

$$
\lim \sup \left(A\left(\left|v_{\varepsilon}\right| \alpha^{\prime-2} v_{\varepsilon}, v_{\varepsilon}\right), v_{\varepsilon}\right) \leqslant(g, v)
$$

So:

$$
\lim \sup \left(A\left(\left|v_{\varepsilon}\right|^{\alpha^{\prime}-2} v_{\varepsilon}, \varphi\right), v_{\varepsilon}-\varphi\right) \leqslant(g, v-\varphi)
$$

for all $\varphi$ in $V$.

It follows from the hypotheses of the theorem that:

$$
\left(g-A\left(|v|^{\alpha^{\prime}-2} v, \varphi\right), v-\varphi\right) \geqslant 0 \quad \text { for all } \varphi \text { in } V \text {. }
$$

Hence:

$$
A\left(|v|^{\alpha^{\prime}-2} v, v\right)=g=f
$$

Set:

$$
u=|v|^{\alpha^{\prime}-2} v .
$$


Then:

$$
u \in L^{\infty}(G) \text { and } v=|u|^{\alpha-2} u \in V
$$

Moreover:

$$
A\left(u,|u|^{\alpha-2} u\right)=f .
$$

The theorem is proved.

3. We shall now give some applications of the theorems proved in the previous section and study the regularity of the solutions. First consider the case when the constraint is in $G$.

THeorem 4. Let $V=W_{0}^{1, p}(G), K=\left\{u: u\right.$ in $L^{p}(G), u \geqslant 0$ a.e. on $\left.G\right\}$ and let $A(u, v)=-\sum_{j=1}^{n} D_{j} A_{j}(x, u, D v)$.

Suppose that:

(i) For each $j, A_{j}(x, u, D v)$ is continuous with respect to $x, u, D v$.

(ii) $\left|A_{j}(x, u, D v)\right| \leqslant C\left\{1+\exp (|u|)+|D v|^{p-1} \exp (|u|)\right\}$.

(iii) $\sum_{j=1}^{n}\left(A_{j}(x, u, D v)-A_{j}(x, u, D w)\right)\left(D_{j} v-D_{j} w\right) \geqslant 0$.

(iv) $\sum_{j=1}^{n} A_{j}(x, u, D u) D_{j} u \geqslant c \sum_{j=1}^{n}\left|D_{j} u\right|^{p}$.

Then for any $f$ in $L^{\infty}(G)$ there exists $u$ in $K \cap V \cap L^{\infty}(G)$ such that:

$$
(A(u, u)-f, v-u) \geqslant 0 \text { for all } v \text { in } V \cap K .
$$

Moreover:

$$
A(u, u) \in L^{\infty}(G)
$$

Proof. (1) $K$ is a closed convex subset of $V$ and $0 \in K$. Let $\beta(v)=-\left|v^{-}\right|^{p-2} v^{-}$where $v^{-}=0$ if $v(x) \geqslant 0$ and $v^{-}=-v(x)$ otherwise. It is easy to check that $\beta$ verifies the hypothesis of Theorem 1. Let $0<\eta<1$ and consider the operator:

$$
A_{1}(u, v)=A(u, v)+\eta u \text {. }
$$

$A_{1}(u, v)$ and $\eta A_{2} v+A_{1}(u, v)$ satisfy all the hypotheses of Theorem 1. Therefore, from Theorem 1, we get:

$$
\eta A_{2} u_{\varepsilon \eta}+A_{1}\left(u_{\varepsilon \eta}, u_{\varepsilon \eta}\right)+\varepsilon^{-1} \beta\left(u_{\varepsilon \eta}\right)=f .0<\eta<\varepsilon<1 .
$$

Moreover, from the proof of Theorem 1 we have:

$$
\eta\left\|u_{\varepsilon \eta}\right\|_{W}^{p+n}+\left\|u_{\varepsilon \eta}\right\|_{V}+\eta\left\|u_{\varepsilon \eta}\right\|_{L^{\infty}(G)} \leqslant C .
$$

$C$ is a constant independent of both $\varepsilon$ and $\eta$.

Since $u_{\varepsilon \eta}$ is in $W$ and hence in $C(\mathrm{cl} G)$ we get:

$$
\operatorname{ess} \underset{\partial G}{\sup }\left|u_{\varepsilon}\right|=0 \text {. }
$$


It follows from Theorem 7.1 of [7], p. 287 that $\left\|u_{\varepsilon \eta}\right\|_{L^{\infty}(G)}$ is majorized by $\left\|u_{\varepsilon \eta}\right\|_{v}, c$, the $L^{\infty}(G)$-norm of $f$.

\section{Hence:}

$$
\left\|u_{\varepsilon \eta}\right\|_{L^{\infty}(G)} \leqslant C \text {. }
$$

$C$ is again a constant independent of both $\varepsilon$ and $\eta$.

(2) We have:

$$
\begin{aligned}
\eta\left(\left(A_{2} u_{\varepsilon \eta},-\left|u_{\varepsilon \eta}^{-}\right|^{s-2} u_{\varepsilon \eta}^{-}\right)\right) & +\left(A_{1}\left(u_{\varepsilon \eta}, u_{\varepsilon \eta}\right),-\left|u_{\varepsilon \eta}^{-}\right|^{s-2} u_{\varepsilon \eta}^{-}\right) \\
& +\varepsilon^{-1}\left\|u_{\varepsilon \eta}^{-}\right\|_{L^{s}+p-2-2(G)}^{s+p-2} \leqslant\|f\|_{L^{\infty}(G)} \mid\left\|u_{\varepsilon \eta}^{-}\right\|_{L^{s+p-2(G)}}^{s-1} \cdot
\end{aligned}
$$

$s$ is any number with $2 \leqslant s<\infty$.

It is not difficult to see that the first two expressions of the left hand side of the above inequality are nonnegative.

Hence:

$$
\varepsilon^{-1}\left\|u_{\varepsilon \eta}^{-}\right\|_{L^{s+p-2}(G)}^{p-1} \leqslant\|f\|_{L^{\infty}(G)} .
$$

Since $u_{\varepsilon \eta}^{-} \in L^{\infty}(G)$, we may let $s \rightarrow+\infty$ and get:

$$
\varepsilon^{-1}\left\|u_{\varepsilon \eta}^{-}\right\|_{L^{\infty}(G)}^{p-1} \leqslant C \text {. }
$$

$C$ is independent of both $\varepsilon$ and $\eta$.

(3) Let $\eta \rightarrow 0$ and the proof of Theorem 1 gives:

$$
A\left(u_{\varepsilon}, u_{\varepsilon}\right)+\varepsilon^{-1} \beta\left(u_{\varepsilon}\right)=f \text {. }
$$

Moreover:

$$
\left\|u_{\varepsilon}\right\|_{L^{\infty}(G)}+\left\|u_{\varepsilon}\right\|_{V}+\varepsilon^{-1}\left\|u_{\varepsilon}^{-}\right\|_{L^{\infty}(G)}^{p-1} \leqslant C .
$$

$u_{\varepsilon}$ is the weak limit in $V$ of $u_{\varepsilon \eta}$ as $\eta \rightarrow 0$.

Let $\varepsilon \rightarrow 0$ then again the proof of Theorem 1 gives:

$$
(A(u, u)-f, v-u) \geqslant 0 \quad \text { for all } v \text { in } K V \text {. }
$$

Since $\varepsilon^{-1} \beta\left(u_{\varepsilon}\right) \rightarrow g$ in the weak*-topology of $L^{\infty}(G)$, we have:

$$
A(u, u)=f-g \text {. }
$$

The theorem is proved.

With some further hypotheses on $A_{j}$, we have a regularity result.

Theorem 5. Let $V, K$ be as in Theorem 4 and let

$$
A(u, v)=-\sum_{j=1}^{n} D_{j} A_{j}(x, u, D v)
$$

Suppose that: 
(i) $A_{j}(x, u, D v)$ is continuously differentiable with respect to $x$, $u$ and $D v$.

(ii) $(1+|D u|)\left(\left|A_{j}(x, u, D u)\right|+\left|\partial A_{j} / \partial u\right|+\left|A_{j k}(x, u, D u)\right|\right)+$ $\left|\partial A_{j} / \partial x_{k}\right| \leqq C\left(1+\exp (|u|)+|D u|^{p-1} \exp (|u|)\right)$.

$$
A_{j k}(x, u, D u)=\partial A_{j}(x, u, D u) / \partial\left(D_{k} u\right) \cdot j, k=1, \cdots, n .
$$

(iii) $\sum_{j, k=1}^{n} A_{j k}(x, u, D v) D_{j} v D_{k} v \geqslant c \sum_{j=1}^{n}\left(1+\left|D_{j} u\right|^{p-2}\right)\left|D_{j} v\right|^{2}$.

Then for any $f$ in $L^{\infty}(G)$, there exists $u$ in $K \cap V \cap L^{\infty}(G)$ such that:

$$
(A(u, u)-f, v-u) \geqslant 0 \quad \text { for all } v \text { in } K V
$$

Moreover:

$$
u \in C^{0, \alpha}(G) \cap C^{1, \lambda}\left(G^{\prime}\right) \cap W^{2, p^{\prime}}(G) .
$$

$0<\alpha, \gamma<1$ and $G^{\prime}$ is any subset of $G$ with $\mathrm{cl} G^{\prime} \subset G$.

Proof. With the above hypotheses on $A_{j}, A(u, v)$ is a semimonotone operator and satisfies all the hypotheses of Theorem 4 . Therefore, there exists $u$ in $K \cap V \cap L^{\infty}(G)$ such that:

$$
(A(u, u)-f, v-u) \geqslant 0 \quad \text { for all } v \text { in } V \text {. }
$$

Moreover:

$$
A(u, u)=f-g \in L^{\infty}(G) .
$$

From the theory of second order elliptic equations, we get:

$$
u \in C^{0, \alpha}(G) \text {. }
$$

E.g. cf. [7] Theorem 1.1, p. 251.

It has been shown by Dubinskii [5] that the solution of $A(u, u)=$ $f-g$ is in $W^{2, p^{\prime}}(G)$ and moreover:

$$
\sum_{j, k=1}^{n} \int_{G^{\prime}}(1+|\operatorname{grad} u|)^{p-2}\left|D_{j} D_{k} u\right|^{2} d x<\infty .
$$

The above relation together with the Hölder-continuity of $u$ gives:

$$
\int_{G^{\prime}}|\operatorname{grad} u|^{p+2} d x<\infty .
$$

$G^{\prime}$ is any subset of $G$ with $\mathrm{cl} G^{\prime} \subset G$.

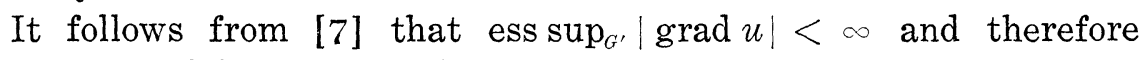
Theorem 6.2 of [7], p. 282 gives:

$$
u \in C^{1,2}\left(G^{\prime}\right) \cap W^{2,2}\left(G^{\prime}\right) .
$$


The theorem is proved.

If the principal part of $A$ is linear, stronger results could be obtained.

Proposition 1. Let $V$ and $K$ be as in Theorem 4 with $p=2$. Let:

$$
A(u, v)=-\sum_{j, k=1}^{n} D_{j}\left(a_{j k}(x) D_{k} v\right)+u \exp (u) .
$$

Suppose that:

(i) $a_{j k} \in C^{2}(\mathrm{cl} G)$.

(ii) $\sum_{j, k=1}^{n} a_{j k} D_{j} v D_{k} v \geqslant c \sum_{j=1}^{n}\left|D_{j} v\right|^{2}$.

Then for any $f$ in $L^{\infty}(G)$ there exists $u$ in $K \cap V \cap W^{2, s}(G)$ such that:

$$
(A(u, u)-f, v-u) \geqslant 0 \quad \text { for all } v \text { in } K V \text {. }
$$

$s$ is any number with $1<s<\infty$.

Proof. We may write

$$
A(u, v)=\mathscr{A} v+u \exp (u) \text {. }
$$

$A(u, v)$ satisfies all the hypotheses of Theorem 4. Thus there exists $u$ in $L^{\infty}(G) \cap K \cap V$ such that:

$$
(A(u, u)-f, v-u) \geqslant 0 \quad \text { for all } v \text { in } K V \text {. }
$$

Moreover:

$$
\mathscr{A} u+u \exp (u)=g \in L^{\infty}(G)
$$

So:

$$
\mathscr{A} u=g-u \exp (u) \in L^{\infty}(G) \text {. }
$$

It follows from the theory of linear elliptic operators that $u \in$ $W^{2,8}(G)$ for any $s$ with $1<s<\infty$.

THEoRem 6. Let $V=W_{0}^{1, p}(G), K=\{u: u$ in $V$, $|\operatorname{grad} u| \leqslant 1$ a.e. on $G$ \} and let $A(u, v)$ be as in Theorem 4. Then for any $f$ in $L^{\infty}(G)$ and for any $\lambda>0$, there exists $u$ in $K \cap L^{\infty}(G)$ such that:

$$
(A(u, u)+\lambda u-f, v-u) \geqslant 0 \text { for all } v \text { in } K \text {. }
$$

Proof. $K$ is a closed convex subset of both $V$ and $W$ with $0 \in K$. Let

$$
\beta(v)=-\sum_{j=1}^{n} D_{j}\left\{\left(1-|\operatorname{grad} v|^{p+n-2}\right)-D_{j} v\right\}
$$


$\beta$ maps bounded sets of $W=W_{0}^{1, p+n}(G)$ into bounded sets of $W^{*}$ and is a monotone hemi-continuous operator. Cf. Lions [9]. Moreover:

$$
((\beta(v), v)) \geqslant\|v\|_{W}^{p+n}-\left(c_{1}+c_{2}\|v\|_{v}^{p+n-2}\right) .
$$

It is not difficult to check that $\beta$ satisfies all the hypotheses of Theorem 2. Thus the result follows from Theorem 2.

Along the lines of Theorem 2, we may consider the case when $K=\left\{u: u\right.$ in $W_{0}^{1, p}(G), u \geqslant 0$ a.e. on $G,|\operatorname{grad} u| \leqslant 1$ a.e. on $\left.G\right\}$. It suffices to apply Theorem 2 with

$$
\beta(v)=-\left|v^{-}\right|^{p-2} v^{-}-\sum_{j=1}^{n} D_{j}\left\{\left(1-|\operatorname{grad} v|^{p+n-2}\right)^{-} D_{j} v\right\} .
$$

We shall now consider the case when constraint imposed on the solution is at the boundary of $G$.

Theorem 7. Let $V=W^{1, p}(G), W=W^{1, p+n}(G), K=\{u$ : $u$ in $V, u \geqslant$ 0 a.e. on $\partial G\}$ and let $A(u, v)$ be as in Theorem 5. Then for any $\lambda>0$ and for any $f$ in $L^{\infty}(G)$ there exists $u \in C^{0, \alpha}(G) \cap C^{1, \mu}\left(G^{\prime}\right) \cap K$ such that:

$$
(A(u, u)-f, v-u) \geqslant 0 \quad \text { for all } v \text { in } K \text {. }
$$

$G^{\prime}$ is any subset of $G$ with $\mathrm{cl} G^{\prime} \subset G$ and $0<\alpha, \mu<1$.

Proof. (1) Let $\beta$ be the penalty operator defined by:

$$
(\beta(v), \phi)=-\int_{\partial G} v-\phi d x, \quad \phi \text { in } V .
$$

$\beta$ satisfies all the hypotheses of Theorem 1. Cf. Lions [9]. Let $0<\varepsilon<1$, then from Theorem 1 we have:

$$
A\left(u_{\varepsilon}, u_{\varepsilon}\right)+\lambda u_{\varepsilon}=f \text { on } G, \partial u_{\varepsilon} / \partial \gamma_{A}=-\varepsilon^{-1} u_{\varepsilon}^{-} \text {on } \partial G .
$$

Moreover, from the proof of Theorem 1 we get:

$$
\left\|u_{\varepsilon}\right\|_{V}+\left\|u_{\varepsilon}\right\|_{L^{\infty}(G)} \leqslant C .
$$

$C$ is a constant independent of $\varepsilon$.

(2) Let $\varepsilon \rightarrow 0$ then:

$$
(A(u, u)+\lambda u-f, v-u) \geqslant 0 \quad \text { for all } v \in K .
$$

Moreover:

$$
A(u, u)+\lambda u=f \text { on } G .
$$


$u$ is the weak limit in $V$ of $u_{\varepsilon}$ as $\varepsilon \rightarrow 0$.

Thus:

$$
A(u, u)=f-\lambda u \in L^{\infty}(G)
$$

Hence:

$$
u \in C^{0, \alpha}(G) \text {. }
$$

A standard argument of the theory of elliptic operators gives:

$$
\sum_{j, k=1}^{n} \int_{G^{\prime}}\left(1+|\operatorname{grad} u|^{p-2}\right)\left|D_{j} D_{k} u\right|^{2} d x<\infty .
$$

$G^{\prime}$ is any subset of $G$ with $\mathrm{cl} G^{\prime} \subset G$.

The Hölder-continuity of $u$ together with the above relation implies that:

$$
\int_{G^{\prime}}|\operatorname{grad} u|^{p+2} d x<\infty
$$

Thus:

$$
u \in C^{1, \mu}\left(G^{\prime \prime}\right) \text { where } \operatorname{cl} G^{\prime \prime} \subset G^{\prime} \subset \operatorname{cl} G^{\prime} \subset G .
$$

The theorem is proved.

\section{REFERENCES}

1. H. Brezis, Equations et inéquations dans les espaces vectoriels en dualité, Ann. Inst. Fourier, 18 (1968), 115-175.

2. —_ Problèmes unilatéraux, J. Math. Pure Appl., 51 (1972), 1-168.

3. H. Brezis and G. Stampacchia, Sur la régularité de la solution d'inéquations elliptiques, Bull. Soc. Math. France, 96 (1968), 153-180.

4. F. E. Browder, Nonlinear monotone operators and convex sets in Banach spaces, Bull. Amer. Math. Soc., 71 (1965), 780-785.

5. Ju. A. Dubinskii, Weak convergence in nonlinear elliptic and parabolic equations, Mat. Sb., 67 (109), (1965), 609-642 = Amer. Math. Soc. Transl., 2 (67), (1968), 226-258.

6. P. Hartman and G. Stampacchia. On some nonlinear elliptic differential functional equations, Acta Math., 115 (1966), 271-310.

7. O. A. Ladyzenskaya and N. N. Uraltseva, Linear and Quasilinear Elliptic Equations, Acad. Press, 1968.

8. H. Lewy and G. Stampacchia, On the regularity of the solution of a variational inequality, Comm. Pure Appl. Math., 22 (1969), 153-188.

9. J. L. Lions, Quelques méthodes de résolution des problemes aux limites non linéaires, Dund. Paris 1969.

10. J. L. Lions and G. Stampacchia, Variational inequalities, Comm. Pure Appl. Math., 20 (1967), 493-519.

Received July 25, 1972.

UNIVERSITY OF BRITISH COLUMBIA 



\section{PACIFIC JOURNAL OF MATHEMATICS}

\section{EDITORS}

RICHARD ARENS (Managing Editor)

University of California

Los Angeles, California 90024

R. A. Beaumont

University of Washington

Seattle, Washington 98105
J. DUGUNDJI*

Department of Mathematics

University of Southern California

Los Angeles, California 90007

D. Gilbarg and J. Milgram

Stanford University

Stanford, California 94305

\section{ASSOCIATE EDITORS}
E. F. BECKENBACH
B. H. NeumanN
F. WOLF
K. YosHIDA

\section{SUPPORTING INSTITUTIONS}

UNIVERSITY OF BRITISH COLUMBIA
CALIFORNIA INSTITUTE OF TECHNOLOGY
UNIVERSITY OF CALIFORNIA
MONTANA STATE UNIVERSITY
UNIVERSITY OF NEVADA
NEW MEXICO STATE UNIVERSITY
OREGON STATE UNIVERSITY
UNIVERSITY OF OREGON
OSAKA UNIVERSITY

UNIVERSITY OF BRITISH COLUMBIA

UNIVERSITY OF CALIFORNIA

MONTANA STATE UNIVERSITY

UNIVERSITY OF NEVADA

OREGON STATE UNIVERSITY

OSAKA UNIVERSITY
UNIVERSITY OF SOUTHERN CALIFORNIA

STANFORD UNIVERSITY

UNIVERSITY OF TOKYO

UNIVERSITY OF UTAH

WASHINGTON STATE UNIVERSITY

UNIVERSITY OF WASHINGTON

\section{AMERICAN MATHEMATICAL SOCIETY} NAVAL WEAPONS CENTER

* C. R. DePrima California Institute of Technology, Pasadena, CA 91109, will replace J. Dugundji until August 1974. 


\section{Pacific Journal of Mathematics}

\section{Vol. 48, No. $1 \quad$ March, 1973}

Jan Aarts and David John Lutzer, Pseudo-completeness and the product of Baire

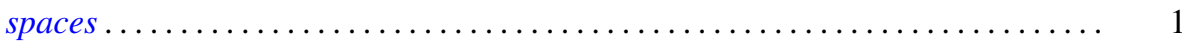

Gordon Owen Berg, Metric characterizations of Euclidean spaces ............ 11

Ajit Kaur Chilana, The space of bounded sequences with the mixed topology ..... . 29

Philip Throop Church and James Timourian, Differentiable open maps of

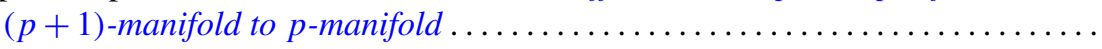

P. D. T. A. Elliott, On additive functions whose limiting distributions possess a finite

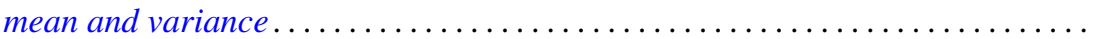

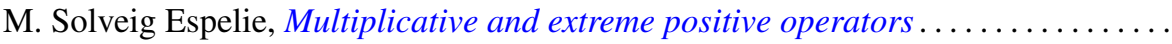

Jacques A. Ferland, Domains of negativity and application to generalized convexity

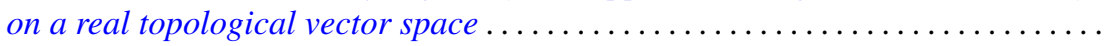

Michael Benton Freeman and Reese Harvey, A compact set that is locally holomorphically convex but not holomorphically convex ...............

Roe William Goodman, Positive-definite distributions and intertwining

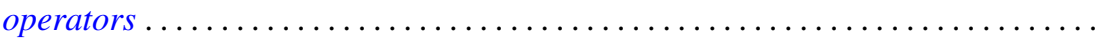

Elliot Charles Gootman, The type of some $C^{*}$ and $W^{*}$-algebras associated with

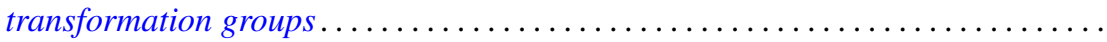

David Charles Haddad, Angular limits of locally finitely valent holomorphic

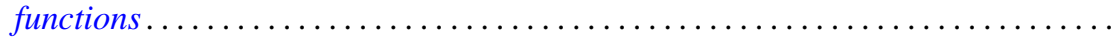

William Buhmann Johnson, On quasi-complements .

William M. Kantor, On 2-transitive collineation groups of finite projective spaces...

Joachim Lambek and Gerhard O. Michler, Completions and classical localizations of right Noetherian rings

Kenneth Lamar Lange, Borel sets of probability measures ......

David Lowell Lovelady, Product integrals for an ordinary differential equation in a Banach space

Jorge Martinez, A hom-functor for lattice-ordered groups .........

W. K. Mason, Weakly almost periodic homeomorphisms of the two sphere ....

Anthony G. Mucci, Limits for martingale-like sequences .......

Eugene Michael Norris, Relationally induced semigroups ...

Arthur E. Olson, A comparison of c-density and $k$-density ......

Donald Steven Passman, On the semisimplicity of group rings of linear groups.

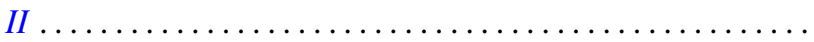

Charles Radin, Ergodicity in von Neumann algebras .

P. Rosenthal, On the singularities of the function generated by the Bergman operator of the second kind.

Arthur Argyle Sagle and J. R. Schumi, Multiplications on homogeneous spaces,

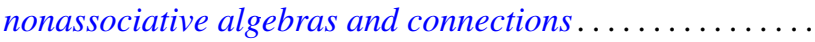

Leo Sario and Cecilia Wang, Existence of Dirichlet finite biharmonic functions on

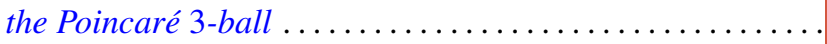

Ramachandran Subramanian, On a generalization of martingales due to Blake ..

Bui An Ton, On strongly nonlinear elliptic variational inequalities.

Seth Warner, A topological characterization of complete, discretely valued

fields. 